\title{
Guest Editorial: Signal Processing for Ultra High Performance and High Efficiency in 5G Mobile Networks
}

\author{
Ta-Sung Lee ${ }^{1}$ Hsuan-Jung $\mathrm{Su}^{2}$
}

Published online: 18 February 2016

(C) Springer Science+Business Media New York 2016

With mobile data traffic growing rapidly in recent years, cellular operators worldwide are facing stringent demands for data volume, system capacity, data rate, traffic offloading, resource management, energy efficiency and response time, and so on. This has motivated the development of the fifth generation $(5 \mathrm{G})$ mobile communications technologies that promise a mobile broadband experience far beyond the current $4 \mathrm{G}$ systems. This special issue aims to provide theoretical and experimental views on key technical issues and challenges to $5 \mathrm{G}$ mobile networks from the signal processing perspective. It contains a selection of research and survey papers in two categories: transmission technologies and resource management.

The first category has six papers with an emphasis on advanced wireless transmission technologies such as multipleinput multiple-output (MIMO) interference channel and massive MIMO precoding, non-orthogonal multiple access (NOMA), millimeter-wave (mmWave) systems, large-scale relay systems, etc.

The paper "Robust Interference Management via Linear Precoding and Linear/Non-Linear Equalization" (10.1007/ s11265-015-1042-0) by Park et al. studies the robust design of linear precoding and linear/non-linear equalization for multi-cell MIMO systems in the presence of imperfect channel state information (CSI). The optimal robust design of linear precoders is tackled for a MIMO interference broadcast

Ta-Sung Lee

tslee@mail.nctu.edu.tw

Hsuan-Jung Su

hjs@ntu.edu.tw

1 National Chiao Tung University, Hsinchu, Taiwan

2 National Taiwan University, Taipei, Taiwan channel (MIMO-IBC) with general unicast/multicast messages in each cell. Then, symbol-by-symbol (non-linear) decision-feedback equalization at the mobile stations (MSs) for a MIMO interference channel (MIMO-IC) is considered with joint optimization of linear precoding and decisionfeedback equalization. Both problems are addressed by proposing iterative algorithms which are shown to be implementable in a distributed fashion on processors that have only local and partial CSI. Numerical results show that the proposed robust solutions significantly improve over conventional non-robust schemes in terms of sum-rate or symbol error rate.

In the paper "Downlink Precoding for Multiple Users in FDD Massive MIMO without CSI Feedback" (10.1007/ s11265-015-1079-0), Tang and Su study downlink precoding for massive MIMO systems in the frequency division duplex (FDD) mode which is usually considered impractical due to the huge amount of pilot signals and feedback involved in obtaining the CSI at the transmitter (CSI-T). By recognizing the similarity between uplink and downlink channels and assuming that partial channel knowledge (specifically, the angles of departure (AoD) of the major propagation paths of each user) is available at the base station, the authors propose two precoding designs based on robust beamforming and spatial-domain optimum finite impulse response (FIR) filter that do not require CSI feedback from the users. Simulation results demonstrate that the proposed method achieves a sum rate near that of a feedback-based precoding method with ideal CSI-T.

The paper "On the Ergodic Capacity and Energy Efficiency for Fading MIMO NOMA Systems" (10.1007/s11265-0151076-3) by Sun et al. explores the potential gains of multiple-input multiple-output (MIMO) non-orthogonal multiple access (NOMA) in terms of ergodic capacity and energy efficiency (EE). The authors study an ergodic capacity 
maximization problem for fading MIMO NOMA systems, and propose a low complexity and near-optimal approach to solving the concerned problem. Numerical results show that the proposed NOMA schemes outperform the traditional orthogonal multiple access scheme in terms of both spectrum and energy efficiency.

The paper "Power Efficient Signal Processing for mmWave $5 G$ Systems" (10.1007/s11265-015-1074-5) by Rajagopal et al. explores the power consumption issue for mmWave $5 \mathrm{G}$ systems and proposes power efficient signal processing architectures and algorithms in order to help mitigate power consumption at both the transmitter and the receiver. Two power efficient signal processing architectures are proposed that efficiently channelize the large bandwidth for low power. Signal processing algorithms are presented that facilitate low power operation in the system by providing dynamic beamwidth support, peak-to-average power ratio reduction, bandwidth adaptation for control and data transmissions and low power channel decoding.

In the paper "Joint AoA and Channel Estimation for SIMOOFDM Systems: a Compressive-sensing Approach" (10.1007/ s11265-015-1043-z), Chen et al. study a joint angle-of-arrival (AoA) and channel estimation problem for mmWave communications systems in the $5 \mathrm{G}$ era. The authors propose novel methods for channel estimation in a SIMO-OFDM system using frequency-domain pilots. With the estimated channels, AoA's are then estimated by the maximum-likelihood method. All in all, this paper provides interesting insights on the design of beamforming and beam tracking techniques for mmWave systems. Simulation results confirm that the proposed method attains the theoretical CRLB.

The paper "Cooperative Transmissions in 5 G Large-Scale Relay Systems: How to Keep a Balance between Performance and Complexity?" (10.1007/s11265-015-1062-9) by Zhao et al. studies the tradeoff between performance and complexity in large-scale decode-and-forward (DF) relay systems. To facilitate asymptotic study, the cumulative distribution function (CDF) of the sum of the $K$-largest order statistics of $N$ independent exponential random variables is developed to obtain explicit expressions for the outage probability and the expected number of relays selected for cooperation. The performance of relay cooperation is also evaluated with the presence of channel estimation error. Numerical results verify the accuracy of the developed theoretic results and also demonstrate the performance and complexity achieved by different relay cooperation strategies.

The second category has six papers with an emphasis on advanced resource management mechanisms such as traffic offloading, ultra-dense small cell deployment, context aware resource allocation, interference control, spectrum sharing, etc.

The paper "On the Traffic Offloading in Wi-Fi Supported Heterogeneous Wireless Networks" (10.1007/s11265-0151064-7) authored by Ekti et al. proposes a new approach to mobile data offloading that can optimize the heterogeneous network traffic. In particular, a user activity and path loss based offloading strategy is presented and compared with the SIR based one. For dense urban settings, hybrid traffictype offloading such as Wi-Fi and D2D are considered. It is shown that substantial improvements can be achieved with the hybrid strategy, allowing for a reduction in the macrocell traffic. Moreover, over-the-air signaling, operational and capital expenditures can be reduced, while spectral efficiency, data rates and coverage can be increased.

The paper "Offloading in Software Defined Network at Edge with Information Asymmetry: A Contract Theoretical Approach" (10.1007/s11265-015-1038-9) by Zhang et al. investigates the incentive problem in software defined network (SDN)-at-edge of how to motivate a third party access points (APs) such as Wi-Fi and smallcells to offload traffic for the central base stations (BSs). Under the information asymmetry that the APs know more about own traffic demands, the BS needs to distribute the payment in accordance with the APs' idle capacity to maintain a compatible incentive. For this problem, a contract theoretic approach is applied to model and analyze the service trading between the BS and APs. In addition, two other incentive mechanisms: optimal discrimination contract and linear pricing contract are introduced to serve as the comparisons of the antiadverse selection contract. Simulation results show that the contract can effectively incentivize APs' participation and offload the cellular network traffic. Furthermore, the antiadverse selection contract achieves the optimal outcome under the information asymmetry scenario.

In the paper "Downward Facing Directional Antennas for Ultra-high Density Indoor Small Cells" (10.1007/s11265015-1034-0), Ho et al. present the concept of deploying very small indoor cells, known as attocells, in higher densities in order to further leverage spatial reuse gains. They address the challenges involved in deploying these very small cells with a coverage range of only a few meters, and propose a novel antenna configuration providing coverage from the ceiling. Extensive simulations are performed indicating that the proposed antenna configuration reduces interference and improves capacity by a factor of 4 compared to using traditional dipole antennas, enabling significant indoor user throughput gains.

The paper entitled "Interference-Robust Air Interface for 5 G Ultra-Dense Small Cells" (10.1007/s11265-015-1060-y) investigates a novel physical layer frame structure optimized for deployment of $5 \mathrm{G}$ ultra-dense small cells operating at cmWave frequencies. Tavares et al. address the usage of victim-aware rank adaptation to manage the inter-cell interference sources. In particular, the Interference Rejection Combining (IRC) receiver is adopted for suppressing intercell interference, while Successive Interference Cancellation (SIC) is used for removing the inter-stream interference. 
System level evaluation shows that the proposed air interface can be a feasible alternative to traditional solutions based on frequency reuse planning.

In the paper "Towards 5G: Context Aware Resource Allocation for Energy Saving" (10.1007/s11265-015-1061$\mathrm{x})$, Alam et al. investigate a context aware scheduling (CAS) algorithm which considers the context information of users, with an aim to achieving a high energy efficiency. The paper provides an information model of context awareness along with a context aware framework for resource management to improve the performance of existing networks toward $5 \mathrm{G}$. System level simulations show that considerable amount of energy is saved by utilizing the proposed CAS compared to conventional scheduling, with a reduction of $10-15 \%$ in UE battery consumption.

In their paper entitled "Detection and Leasing of Joint Space and Spectrum Opportunities by Multiple Secondary Network Operators in Cognitive Radio Systems" (10.1007/s11265015-1085-2), Li et al. explore how to allocate or lease space and spectrum opportunities to the secondary users in a cognitive network. They present models of detection probability of the primary users at overlapped beams and spectrum leasing market for the secondary users to opportunistically access the temporarily unused resource in the framework of differential games. The efficacy of the proposed methods is confirmed by computer simulations.

This special issue presents a comprehensive technical perspective of the fundamentals and advancements of ultra high performance and high efficiency design in 5G mobile networks. We hope you find this special issue informative and helpful. We thank all the authors for their contributions, and all the anonymous reviewers for their valuable comments. We also extend our gratitude to the team at Springer for their high quality support.

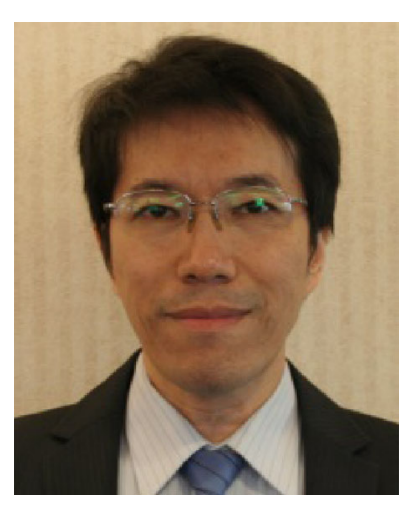

Ta-Sung Lee received the B.S. degree from National Taiwan University in 1983, the M.S. degree from University of Wisconsin, Madison, in 1987, and the Ph.D. degree from Purdue University, W. Lafayette, IN, in 1989, all in electrical engineering. In 1990, he joined the Faculty of National Chiao Tung University (NCTU), Hsinchu, Taiwan, where he holds a position as Professor of Department of Electrical and Computer Engineering. From 1999 to 2001 , he was Director of Communications \& Computer Continuing Education Program, NCTU. From 2005 to 2007, he was Chairman of Department of
Communication Engineering, and from 2007 to 2008 and 2012-2013, he was Dean of Student Affairs of NCTU. From 2008 to 2010, he was Commissioner of the National Communications Commission (NCC), a regulatory agency of Taiwan similar to the FCC, and responsible for the strategic planning, policy making and technical regulation for the telecommunications and broadcasting services. He was Vice Chairman and Chairman of IEEE Communications Society Taipei Chapter for 20052008, a Board Member of IEEE Taipei Section for 2007-2010, an Associate Editor of IEEE Transactions on Signal Processing for 20092013, and IEEE Signal Processing Society Regional Director-at-Large for R10 for 2011-2013. He is currently an Area Editor of Journal of Signal Processing Systems. He has been Chairman of Telecom Technology Center, a government funded agency for telecommunications R\&D, since 2013. Dr. Lee is actively involved in research and development in signal processing and system design for wireless communications. He is an IEEE Fellow, and has won several awards for his research, engineering and education contributions; these include Young Electrical Engineer Award of the Chinese Institute of Electrical Engineering (CIEE), Distinguished Electrical Engineering Professor Award of CIEE, NCTU Distinguished Scholar Award, and NCTU Teaching Award.

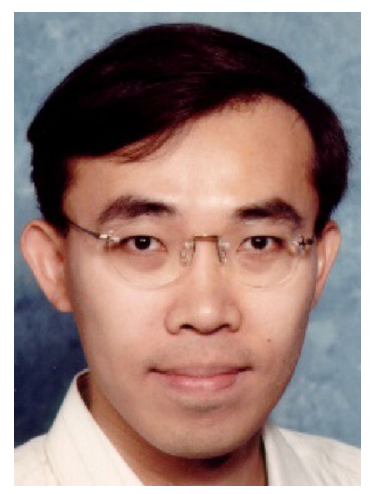

Hsuan-Jung Su received the B.S. degree in Electronics Engineering from the National Chiao Tung University, Taiwan, in 1992, and the M.S. and Ph.D. degrees in Electrical Engineering from the University of Maryland, College Park, in 1996 and 1999, respectively. From 1999 to 2000 , he was a Postdoctoral Research Associate with the Institute for Systems Research, University of Maryland. From 2000 to 2003, he was with the Bell Laboratories, Lucent Technologies, Holmdel, New Jersey, where he received the Central Bell Labs Teamwork Award in 2002 and the Bell Labs President's Gold Award in 2003 for his contribution to the $3 \mathrm{G}$ wireless network design and standardization. In 2003, Dr. Su joined the Department of Electrical Engineering and Graduate Institute of Communication Engineering, National Taiwan University, where he is currently a Professor. From 2014 to 2015, Dr. Su was a Visiting Fellow at Princeton University. Dr. $\mathrm{Su}$ is an Area Editor of the Physical Communication (PHYCOM) journal (Elsevier), and has guest edited special issues for journals such as IEEE Access. He has also served on the organizing committees and TPCs of many international conferences. He served as the Finance Chair of IEEE ICASSP 2009, the Finance Co-Chair and a TPC Track Chair of IEEE VTC 2010 Spring, a TPC Co-Chair of WPMC 2012, a TPC Co-Chair of IEEE GreenCom 2014, and a TPC Co-Chair of WOCC 2015. Dr. Su was the Chair of IEEE Information Theory Society, Taipei Chapter (20132015), and the Secretary and Treasurer of the IEEE Communications Society Asia-Pacific Board (2014-2015). His research interests cover coding, modulation, signal processing, synchronization, interference management, resource allocation, and MAC protocols of wireless communication, cognitive, M2M and D2D networks. 